\title{
An Integrated Approach to Modeling Solar Electric Propulsion Vehicles During Long Duration, Near-Earth Orbit Transfers
}

\author{
David A. Smith* \\ Vantage Parters LLC, Cleveland, OH, 44135, U.S.A. \\ Jeffrey S. Hojnicki ${ }^{\dagger}$ and Waldy K. Sjauw ${ }^{\ddagger}$ \\ NASA Glenn Research Center, Cleveland, OH, 44135, U.S.A.
}

\begin{abstract}
Recent NASA interest in utilizing solar electronic propulsion (SEP) technology to transfer payloads, e.g. from low-Earth orbit (LEO) to higher energy geostationary-Earth orbit (GEO) or to Earth escape, has necessitated the development of high fidelity SEP vehicle models and simulations. These models and simulations need to be capable of capturing vehicle dynamics and sub-system interactions experienced during the transfer trajectories which are typically accomplished with continuous-burn (potentially interrupted by solar eclipse), long duration "spiral out" maneuvers taking several months or more to complete. This paper presents details of an integrated simulation approach achieved by combining a high fidelity vehicle simulation code with a detailed solar array model. The combined simulation tool gives researchers the functionality to study the integrated effects of various vehicle sub-systems (e.g. vehicle guidance, navigation and control (GN\&C), electric propulsion system (EP)) with time varying power production.

Results from a simulation model of a vehicle with a $50 \mathrm{~kW}$ class SEP system using the integrated tool are presented and compared to the results from another simulation model employing a $50 \mathrm{~kW}$ end-of-life (EOL) fixed power level assumption. These models simulate a vehicle under three degree of freedom dynamics (i.e. translational dynamics only) and include the effects of a targeting guidance algorithm (providing a "near optimal" transfer) during a LEO to near Earth escape $\left(C_{3}=-2.0 \mathrm{~km}^{2} \mathrm{sec}^{-2}\right)$ spiral trajectory. The presented results include the impact of the fully integrated, time-varying solar array model (e.g. cumulative array degradation from traversing the Van Allen belts, impact of solar eclipses on the vehicle and the related temperature responses in the solar arrays due to operating in the Earth's thermal environment, high fidelity array power module, etc.); these are used to assess the impact on vehicle performance (i.e. propellant consumption) and transit times.
\end{abstract}

*Aerospace Engineer, Mission Design and Analysis Branch, david.a.smith-1@nasa.gov, AIAA Member.

$\dagger$ Electrical Engineer, Power Architecture and Analysis Branch, jeffrey.s.hojnicki@nasa.gov

$\ddagger$ Aerospace Engineer, Mission Design and Analysis Branch, waldy.k.sjauw@nasa.gov 


\section{Nomenclature}

$\begin{array}{ll}\text { argp } & \text { Argument of perigee, deg } \\ e c c & \text { Eccentricity } \\ C 3 & \text { Characteristic energy, } \mathrm{km}^{2} \mathrm{sec}^{-2} \\ g & \text { Earth gravitational constant, } \mathrm{sec}^{-2} \\ \text { inc } & \text { Inclination, deg } \\ \text { raan } & \text { Right ascension of the ascending node, deg } \\ \text { sma } & \text { Semi-major axis, } \mathrm{km} \\ \text { trua } & \text { True anomaly, deg } \\ I_{\text {sp }} & \text { Specific impulse, sec } \\ I V & \text { Current-voltage (curve) } \\ I_{@ V_{\text {cost }}} & \text { Current associated with a specific constant voltage, amp } \\ P_{\text {eng }} & \text { Engine power, watt } \\ V_{\text {cnst }} & \text { Constant voltage, volt } \\ 3 D O F & \text { Three-Degree of Freedom } \\ 6 D O F & \text { Six-Degree of Freedom } \\ B O L & \text { Beginning of Life (array power assumption) } \\ D A G & \text { Directional Adaptive Guidance } \\ E O L & \text { End of Life (array power assumption) } \\ G E O & \text { Geosynchronous Earth orbit } \\ I S S & \text { International Space Station } \\ L E O & \text { Low Earth orbit } \\ M A S T I F & \text { Mission Analysis Simulation Tool in Fortran } \\ P P U & \text { Power Processing Unit } \\ R C S & \text { Roll Control System } \\ S E P & \text { Solar Electric Propulsion } \\ S E P S i m & \text { Solar Electric Propulsion Simulation (tool) } \\ S P A C E & \text { System Power Analysis for Capability Evaluation } \\ \beta_{\text {sun }} & \text { Solar beta angle, deg } \\ \eta_{\text {eff }} & \text { Engine efficiency } \\ & \end{array}$

\section{Introduction}

T TRADITIONALLY, most spacecraft that primarily use solar electric propulsion (SEP) have operated entirely in interplanetary space where predicting the performance of the solar arrays is straightforward. Since sun-pointing is easily achieved, solar array degradation mechanisms operate on time scales of months or years, and since there are no, or few, eclipse periods, solar array power, can be estimated as a fixed value that only changes sporadically, primarily when the vehicle moves further from or closer to the sun. Recent NASA interest in utilizing SEP technology to transfer payloads (e.g. from low-Earth orbit (LEO) to higher energy geostationary-Earth orbit (GEO), to Earth escape, or onto lunar or Martian trajectories) has necessitated the development of high fidelity SEP vehicle models and simulations. These spiral shaped trajectories induce significantly different environments on spacecraft compared to interplanetary transfer trajectories.

From a power generation viewpoint, these high fidelity models must capture effects, which cause solar array performance to change with time. Solar array temperatures vary significantly within orbits, impacting array power and current-voltage characteristics. Perfect solar pointing may not be achieved, as changing solar beta angles $\left(\beta_{\text {sun }}\right)$ require multi-axis solar array gimbals, or attitude steering maneuvers to maintain excellent pointing ${ }^{1}$ and thus might not be implemented. Long traversals through the Van Allen radiation belts significantly degrade solar array performance, up to $20 \%$ or more, depending on the solar array design and the time spent in the belts. To capture these significant effects on the power available to the thrusters, a more sophisticated power system model is required. Because the power produced affects the trajectory, which then, in turn, affects the power produced, the power model must be integrated within the trajectory simulation to accurately account for all the synergistic effects.

From a trajectory design viewpoint, the SEP vehicle models and simulations must capture vehicle dynam- 
ics and sub-system interactions experienced during the transfer trajectories which are typically accomplished with continuous-burn (potentially interrupted by solar eclipse), long duration "spiral out" maneuvers taking several months or more to complete. As previously mentioned, the slow transfer from LEO, unlike deep space SEP missions, presents unique challenges to SEP simulations, in that while the vehicle is operating at relatively lower altitudes it can experience frequent sun/eclipse crossings and a varying near-Earth radiation environment associated with the Van Allen belts, as mentioned above. Current, state-of-the-art, techniques for studying SEP transfer problems typically utilize multiple decoupled subsystem models to study individual vehicle components and independently compare results to refine the study. This process is often time consuming and requires simplifying each subsystem as contributions from other, coupled, subsystems are ignored. Besides the compromise in fidelity, this approach is also iterative and highly susceptible to human error.

\section{Existing Vehicle Simulation and Solar Array Models and the Combined SEPSim Tool}

To address the above-mentioned issues associated with modeling SEP vehicles during transfers near Earth, the authors discuss the development of a rigid body vehicle simulation tool configured to incorporate guidance, navigation and control (GN\&C) with a high fidelity solar array model and electric propulsion (EP) system models. This tool interfaces and "merges" an existing, high fidelity vehicle simulation code, Mission Analysis Simulation Tool in Fortran (MASTIF), ${ }^{2}$ with modules from an existing detailed power system model, System Power Analysis for Capability Evaluation (SPACE). ${ }^{3}$ MASTIF is a simulation tool developed at NASA Glenn Research Center (GRC) and provides a modular platform for simulating key vehicle dynamics and is responsible for integrating the vehicle's position and attitude in the combined tool. SPACE, also developed at GRC, is a high fidelity spacecraft power system model. These are combined with EP thruster models to comprise the overall mechanism for advancing the vehicle state. The combined tool is called Solar Electric Power Simulation (SEPSim). The combination of robust and high fidelity dynamics, array and EP models, along with their interactions (i.e. coupled effects), into one simulation tool eliminates the need for individual subsystem models, thus providing a much more detailed, efficient and less error prone methodology for studying SEP vehicles. The two original codes and the merger into SEPSim will be briefly discussed and detailed in this section of the paper.

\section{A. Vehicle Simulation Tool (MASTIF)}

MASTIF $^{2}$ is a low to high fidelity vehicle simulation tool written primarily in Fortran 90/95/03. MASTIF is designed, written and maintained at NASA GRC; originally, under funding from the Ares I project as a test bed for GN\&C algorithms and to provide independent verification and validation of ascent trajectories generated by other NASA simulation tools ${ }^{4}$ at other NASA centers. MASTIF may be configured to simulate three degree of freedom (3DOF) translational dynamics only, or in higher fidelity mode to produce full six degree of freedom (6DOF) vehicle simulations capturing both translational and rotational dynamics. In addition to Ares I work, MASTIF has been used to simulate launch and in-space vehicles and missions such as the MARS Ascent Vehicle, ${ }^{5}$ Earth Departure Stage loiter and various "in-house" SEP-Technology Demonstration Mission low thrust mission and vehicle design studies.

MASTIF incorporates a wide variety of subsystem models including, but not limited to: a suite of GN\&C algorithms, engine/thruster models, mass property models, gravity models and various atmosphere models and drag formulations for launch vehicle problems. Simulations can be produced for launch vehicles originating on ground-based launch pads as well as for in-space vehicles where initial conditions are specified via orbital parameters. The code is designed taking advantage of Fortran 90/95/03 class structure under a highly modular architecture. The core of MASTIF provides the user a choice of integration engines, coordinate systems and transformations, math libraries, event processing and a robust user interface. In general, sub-systems in MASTIF are implemented as self-contained modules, which interact and "communicate" with the main body of MASTIF through well-defined application programmer interfaces. As such, MASTIF provides an excellent platform for independent subsystem designers and experts to "plug" in their existing (or independently derived) subsystem models. This way MASTIF's core functionality is increased without requiring the subsystem experts to understand, or concern themselves with, the details of other vehicle dynamics, subsystems and the vehicle simulation itself. To date, the best example of this extension 
to MASTIF is the inclusion of several modules, from SPACE, to form SEPSim.

\section{B. Solar Power Model (SPACE)}

To predict the solar array performance, SEPSim implements the photovoltaic performance modules from SPACE. ${ }^{3}$ SPACE is a detailed end-to-end spacecraft power system model that was originally developed to model the International Space Station (ISS) power system. Because the algorithms and performance models in SPACE have been validated against on-orbit telemetry, ${ }_{6}^{6}$ SEPSim is able to re-use existing, validated modules without the need to develop new ones from scratch. The solar array modules incorporated from SPACE include: 1) solar array pointing algorithms, which can provide full or limited tracking of the sun, 2) thermal models that predict array temperature accounting for solar and planetary interactions, 3) geometric models to predict solar array shadowing from the spacecraft, and 4) electrical performance models that predict solar array current-voltage characteristics as a function of temperature, off-pointing, solar flux, albedo flux, shadowing and degradation mechanisms, including radiation. Although developed for ISS, SPACE is able to model modern solar arrays and cells, and has been used extensively in support of the Orion Multi-Purpose Crew Vehicle. ${ }^{7}$

In addition to the solar array modules, SEPSim also incorporates battery performance modules. These can be used to account for the energy needed to supply vehicle housekeeping power during eclipse periods, and the corresponding amount of energy needed from the solar array to restore battery charge during insolation. Modules that determine losses in wiring between the solar array and the EP thrusters are also included, since those losses become more significant at the power levels being considered for cargo transport.

The SPACE modules also include direct ties to the AE8/AP8 8 radiation models, so that radiation fluence, and its associated impact on solar array performance, can be predicted by the model in-situ. This allows SEPSim to degrade the solar arrays with time during the belt transit, which is a significant improvement to previous modeling methods, which would either assume a constant power level available during the entire scenario, or at best degrade the array performance in periodic steps (typically months in duration) that are estimated in an off-line calculation.

\section{SEPSim Combined Tool Design}

Figure 1 is a schematic overview of SEPSim and illustrates the interface between MASTIF and SPACE. While the details and the specifics of the numerical approach which underlies the code merger is beyond the scope of this paper, the interface can be summarized as follows: given an initial vehicle state, MASTIF integrates the vehicle forward in time. MASTIF then passes the resulting position and velocity $(\vec{R}$ and $\vec{V})$ (and orientation if the simulation is $6 \mathrm{DOF}$ ), as well as the vehicle's relative position to the sun $\left(\vec{R}_{\text {sun }}\right)$ to the power module. The power module then uses this information to point the arrays for power generation under all of the assumptions and options discussed in Section II.B. Although it isn't necessary for the power module to point the arrays in such a way to generate maximum power (user configurable through input), it is often the desired algorithm and the only option discussed in this paper. The power module then calculates an instantaneous current-voltage $(I V)$ curve, which is returned to the MASTIF side of SEPSim. The power module is highly configurable and is able to account for all the effects and impacts on power generation detailed in the previous section. The power module is able to return, at the user's discretion, pure $I V$ curves (such as would be used in a direct-drive model) or, alternatively, simulate the effects of pre-conditioning the $I V$ curves with a built in Power Processing Unit (PPU) model. The resulting power curves are then interrogated by the vehicle simulation side of the code under an assumption of: 1) constant voltage (i.e. constant $I_{s p}$ ), 2) constant current (i.e. constant mass flow rate) or 3) peak power. The resulting power level is then supplied to the EP module where it is converted to thrust. The resulting thrust is used to advance the vehicle forward in time and the process repeats. As with all other subsystems in MASTIF, the user may specify the call frequency for the power model thus activating the power interface as frequently as once per integration time step, or any multiple there of.

The overall benefit of the SEPSim implementation in MASTIF is that of being able to model, with a high degree of fidelity, an SEP vehicle including the integrated impact of one its main subsystems, the solar arrays used for power generation. The resulting trajectory has multiple benefits including (but not limited to): a transfer path that takes into account multiple real life impacts on the power generation plant, the subsequent impact on the EP system, detailed impacts on the solar arrays as a result of the in-situ conditions 
and, thus, on the subsequent path followed. Unlike previous decoupled approaches, all interactions of the various vehicle subsystems are accounted for making the results much more accurate and valuable.

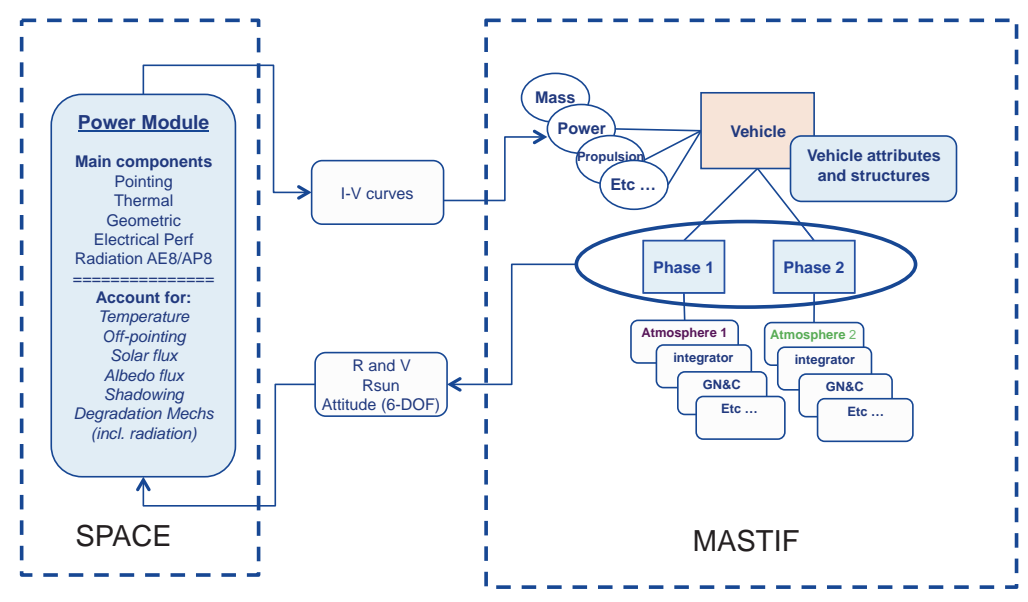

Figure 1: Block diagram of the SEPSim, MASTIF to SPACE interface

\section{Sample SEPSim Cases}

This paper focuses on two notional Earth escape problems to illustrate SEPSim functionality. The first case is a comparison of simulating a $50 \mathrm{~kW}$ class vehicle as it spirals from LEO to near Earth escape under a traditional end-of-life (EOL) power assumption and, alternatively, using the fully integrated power module of SEPSim (Figure 2). This simplified example of SEPSim's capability demonstrates differences in the power profile for the two simulations and how including the detailed, time-varying, power model could potentially be used by the mission designer to shorten mission trip time (amongst others). This case is also used to point out some of the features of the power code such as array temperature impact on power (and therefore thrust) as the vehicle transits in and out of the Earth's shadow. The second set of simulations is intended to illustrate the impact of including roll steering (GN\&C modules) on the time history of the power system. This second comparison is an indication of the types of studies that are difficult to do without the combined SEPSim tool.
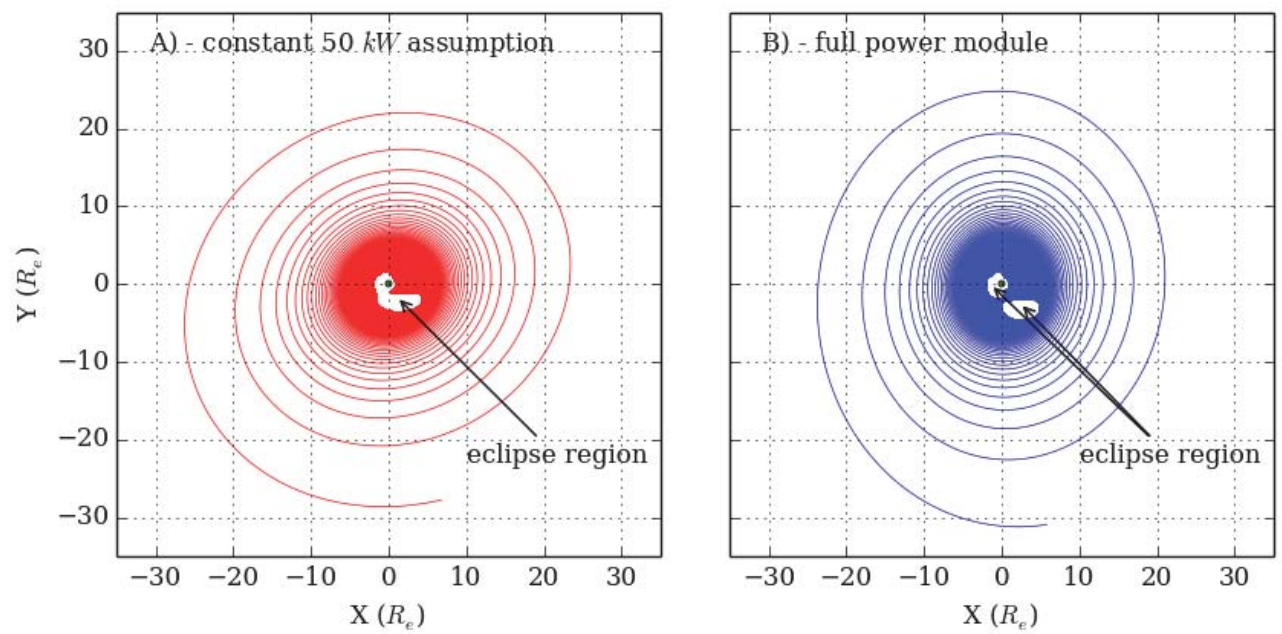

Figure 2: $X-Y$ plots for LEO to near Earth escape spiral tfor: A) $50 \mathrm{~kW}$ assumption (red), and B) full power module (blue). $X$ and $Y$ axes are in EME2K and scaled by Earth radius (Re). Earth location is indicated with a green circle (not to scale) while Earth eclipse regions are illustrated with white space. 
Table 1 provides an overview of initial and targeting/ending conditions as well as other key simulation parameters. Unless otherwise noted, indicated parameters are common to all simulations. In general, all simulations are those of a $6500 \mathrm{~kg}, 50 \mathrm{~kW}$ SEP vehicle during a spiral from LEO to near Earth escape and use MASTIF's Directional Adaptive Guidance (DAG) ${ }^{9}$ to target a large final sma thus spiraling the vehicle into increasingly higher orbits; however, the final termination criterion is satisfied when the vehicle reaches near escape conditions (Table 1). With the exception of the zero roll case in Section III.B, the vehicle is roll-oriented to provide perfect sun pointing of the solar arrays. Effects of Earth shadow ${ }^{10}$ are included and have a direct impact on the solar arrays. ${ }^{3}$ There is no thrusting while the vehicle is in shadow.

Table 1: Initial/Target Conditions \& Simulation Parameters

\begin{tabular}{|c|c|c|}
\hline \multicolumn{3}{|c|}{ Initial and Targeting Conditions } \\
\hline & Initial Conditions & Target Conditions \\
\hline $\operatorname{sma}(\mathrm{km})$ & $6778($ alt $=400)$ & $200 k$ (terminated near escape) \\
\hline$e c c$ & 0 & none \\
\hline inc (deg) & 28.5 & none \\
\hline raan (deg) & 0 & none \\
\hline $\operatorname{argp}(\mathrm{deg})$ & 180 & none \\
\hline truea (deg) & 0 & none \\
\hline mass $(\mathrm{kg})$ & 6500 & NA \\
\hline epoch (UTC) & 2015 Mar 15 00:00:0.00 & NA \\
\hline \multicolumn{3}{|c|}{ Simulation Details } \\
\hline array power class & \multicolumn{2}{|c|}{$50 \mathrm{~kW}$ class (details vary per simulation) } \\
\hline$G N E C$ & \multicolumn{2}{|c|}{ Directional Adaptive Guidance (pitch and yaw) } \\
\hline roll steering & \multicolumn{2}{|c|}{ perfect pointing (unless otherwise noted) } \\
\hline integration time step & \multicolumn{2}{|r|}{$60 \mathrm{sec}$} \\
\hline subsytem freq (incl. power) & \multicolumn{2}{|r|}{$60 \mathrm{sec}$} \\
\hline Earth Shadow (On/Off) & \multicolumn{2}{|r|}{ On } \\
\hline Atmosphere Model & \multicolumn{2}{|r|}{ none } \\
\hline Fidelity Level & \multicolumn{2}{|r|}{$3 \mathrm{DOF}$} \\
\hline Propulsion Model & \multicolumn{2}{|c|}{ Standard EP equation $\left(\eta_{e f f}=65 \%\right.$ and $\left.I_{s p}=3000 s e c\right)$} \\
\hline PPU Model (Yes/No) & \multicolumn{2}{|c|}{ Yes $\left(P P U_{e f f}=95 \%\right)$} \\
\hline Simulation Termination & \multicolumn{2}{|c|}{ Earth Escape $\left(C 3=-2.0 \mathrm{~km}^{2} \mathrm{sec}^{-2}\right)$} \\
\hline
\end{tabular}

\section{Propulsion Module}

The power model results of SEPSim (i.e. SPACE) can be returned directly (direct drive mode) or, as in the case of the results in this paper, through a PPU. The resulting $I V$ curves are interrogated in the propulsion module under a constant voltage assumption of $V_{\text {cnst }}=300 \mathrm{v}$ and the corresponding current $\left(I_{@} V_{c n s t}(a m p)\right)$ is then used to calculate engine/thruster power.

$$
P_{\text {eng }}=I_{\left(V_{\text {cnst }}\right)} \cdot V_{\text {cnst }} \quad(\text { watt })
$$

Detailed propulsion models can be included in SEPSim much the same way the power module is included; however, the results presented in this paper, $P_{\text {eng }}$, are simply used in the standard EP equation to calculate thrust.

$$
\text { Thrust }=\frac{2 \cdot \eta_{e f f} \cdot P_{e n g}}{g \cdot I_{s p}} \quad(N)
$$

\section{A. Problem 1: Assumed $50 k W$ Versus Power Module}

A common practice in mission and power array design is to take a non-integrated approach to solve the problem. In such an approach, a simulation model assumes a constant power level from a solar array that is still available at the array's EOL. The resulting trajectory and assumptions are subsequently passed to an array design team that oversize the solar arrays to guarantee the EOL assumption throughout the 
simulation. Any mismatch between the mission and the array design is then resolved by iterating (manually) on this process until satisfactory results are achieved. While conservative, this approach is less accurate, time consuming and subject to human error.

To illustrate a potential advantage of using the integrated approach available through SEPSim, a notional mission is considered using a $50 \mathrm{~kW}$ class SEP (designed to deliver approximately $50 \mathrm{~kW}$ power at EOL) employing the fully time varying power system (Table 1). In an idealized assumption, any excess power initially generated, due to oversized arrays, is assumed to be available for thrust in Equations 1 and 2. This assumption would require an EP thruster with variable set points (and would most likely not be congruent with the assumption of a constant $I_{s p}$ ), or, alternatively, the presence of additional, spare, thrusters to take advantage of excess power; such specific vehicle details are not relevant for the purpose and scope of this example. The results of this simulation are then compared to the results of a typical $50 \mathrm{~kW}$ EOL assumption where no such excess power is ever available.

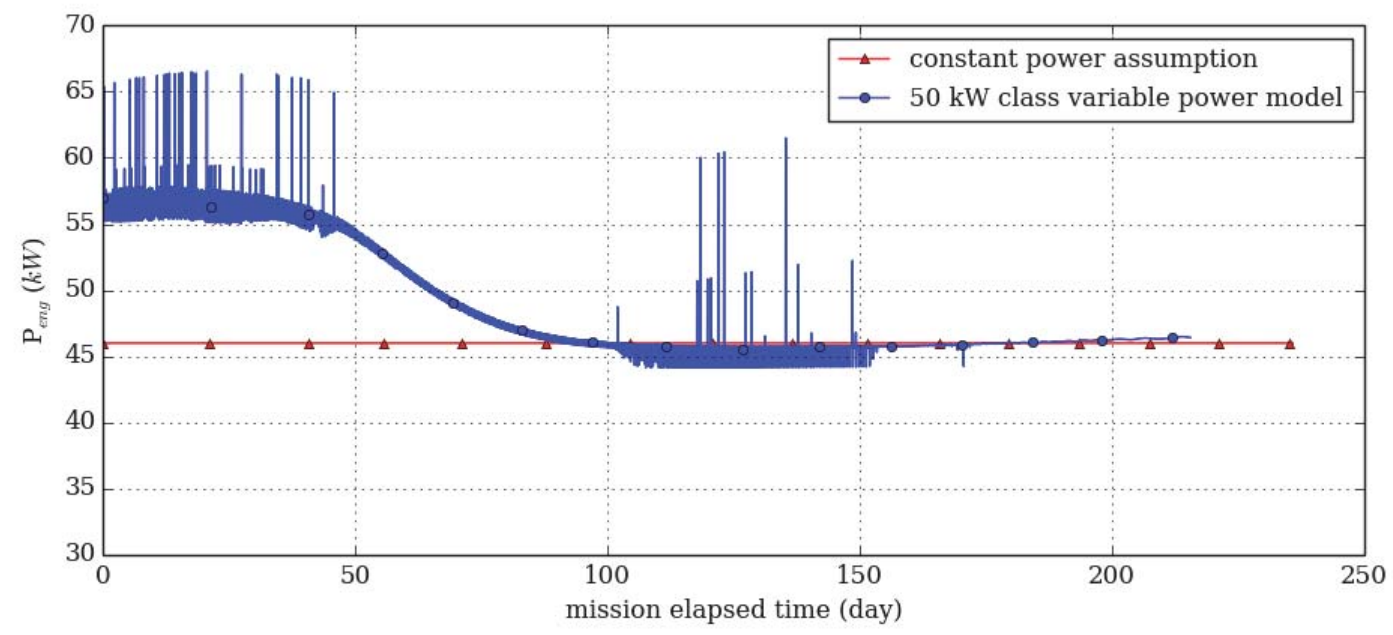

Figure 3: Engine power $(\mathrm{kW})$ as a function of mission time (days). Constant $50 \mathrm{~kW}$ EOL assumption is illustrated in red while the fully time varying power case is illustrated in blue. For plotting clarity, the zero power conditions during eclipse have been suppressed. Note: results also reflect 95\% PPU efficiency (Table 1)

\section{Mission Response to Power Generation}

Figure 3 illustrates the time history of power generation for the constant EOL and time varying power model. The red line represents the simplified model, where the solar array power is set to a constant $50 \mathrm{~kW}$. Note that the power shown is approximately $47.5 \mathrm{~kW}$ because the plot shows thruster input power and thus accounts for losses in the $95 \%$ efficient PPU. The blue curve shows solar array power determined from the more detailed SPACE modules, where the array is represented as a series/parallel combination of individual solar cells and associated interconnects and wiring, selected to produce approximately $50 \mathrm{~kW}$ at the end of the mission. In that model, the power is affected by changes in temperature, solar flux and degradation due to radiation and other factors, and so the EOL power may not exactly match the $50 \mathrm{~kW}$ sizing goal. The PPU efficiency is also applied to the blue curve. The resulting spirals for the two simulations are illustrated in Figure 2 while the time history of key simulation parameters are presented in Figure 4.

The impact of solar array degradation can easily be seen in Figure 3 (detailed subsection can be seen in Figure 5). To achieve a $47.5 \mathrm{~kW}$ power level at the end of the spiral, the solar array must be sized to produce approximately $57 \mathrm{~kW}$ at beginning-of-life (BOL) (not accounting for short term peaks which are discussed below), which offsets the radiation degradation during the spiral. Nearly all of the radiation degradation happens between 50 and 100 days into the mission, which is during the traversal of the proton belt. Passage through the outer electron belts induces significantly less degradation, and a small amount of power is regained as the solar arrays operate cooler at increasing altitudes. The detail produced by this model is something that is not captured in "real-time" during a typical, non-integrated mission/array design approach.

In this notional problem the arrays are oversized at the beginning of the simulation in order to satisfy the $50 \mathrm{~kW}$ EOL assumption. The excess power is converted to thrust the effects of which can be seen in 

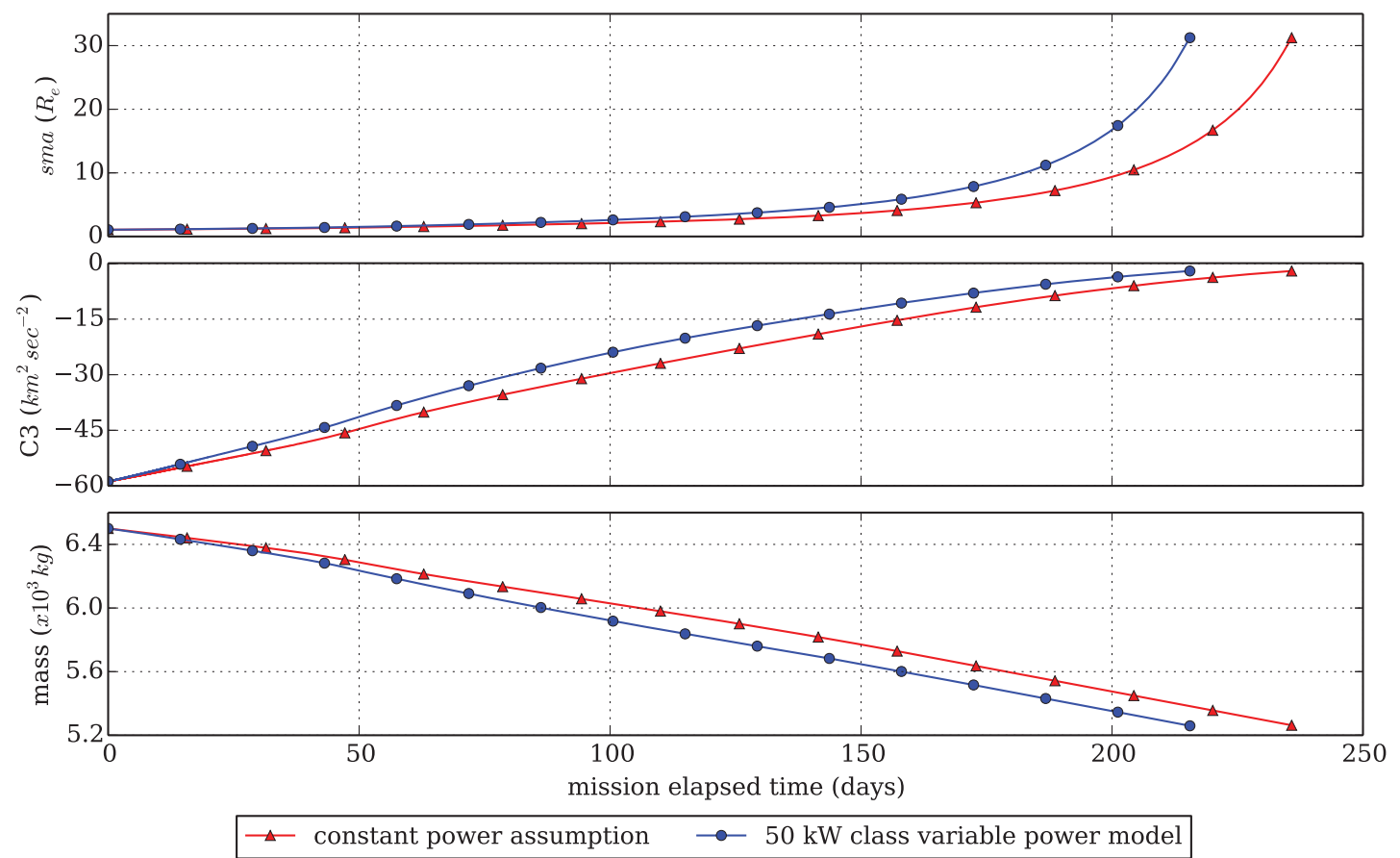

Figure 4: Time history of sma, C3 and vehicle mass for the LEO to near Earth escape spiral out trajectories. Red represents the time history for the $50 \mathrm{~kW}$ EOL assumption while blue indicates the time history for the full time varying power model.

Figure 4 as a shortening of overall mission duration by approximately 2 weeks. Such timesaving could be important if it is pertinent to the payload (e.g. a commercial interest). In reality, the excess power at the beginning of the mission may not all be available for thrust. The mission and array designers may use the excess power to offset array-pointing losses to save RCS propellant or to charge batteries. It should be noted that under the limiting assumption of constant $I_{s p}$, this comparison results in shorter duration missions without a significant (if any) savings in propellant as seen in the vehicle mass comparison. In reality, mission and EP system designers would trade variable thrust and $I_{s p}$ to address this issue. Alternatively, using the BOL power could allow the solar array to be made smaller, while still achieving other mission goals, which could result in substantial cost savings for the mission. Such an analysis is beyond the scope of this paper.

\section{Thermal Response on Power Generation}

While the overall array degradation in response to the radiation environment can easily be seen in Figure 3 a higher frequency fluctuation in power generation is also obvious. When examining a small subsection of the $P_{\text {eng }}$ plot and comparing it to computed array temperature (Figure 5) one can see the impact of the power system thermal model on power generation (and subsequently on thrust and its impact on the mission). The thermal model in the power module predicts array temperature based on exposure to the sun, the Earth's thermal budget and power generation itself. As the SEP enters Earth shadow, the arrays cool down (and also produce zero power). When emerging from shadow the arrays are at their coldest and when they start generating power produce brief power peaks of up to $10 \mathrm{~kW}$ above the continuous power levels for a few minutes. The extra energy available from these transient increases may or may not be usable, depending on the design of the EP system, but would have to be dealt with in a direct-drive system. These features are another example of the type of information that is available when using the integrated SEPSim tool over traditional, independent studies. 


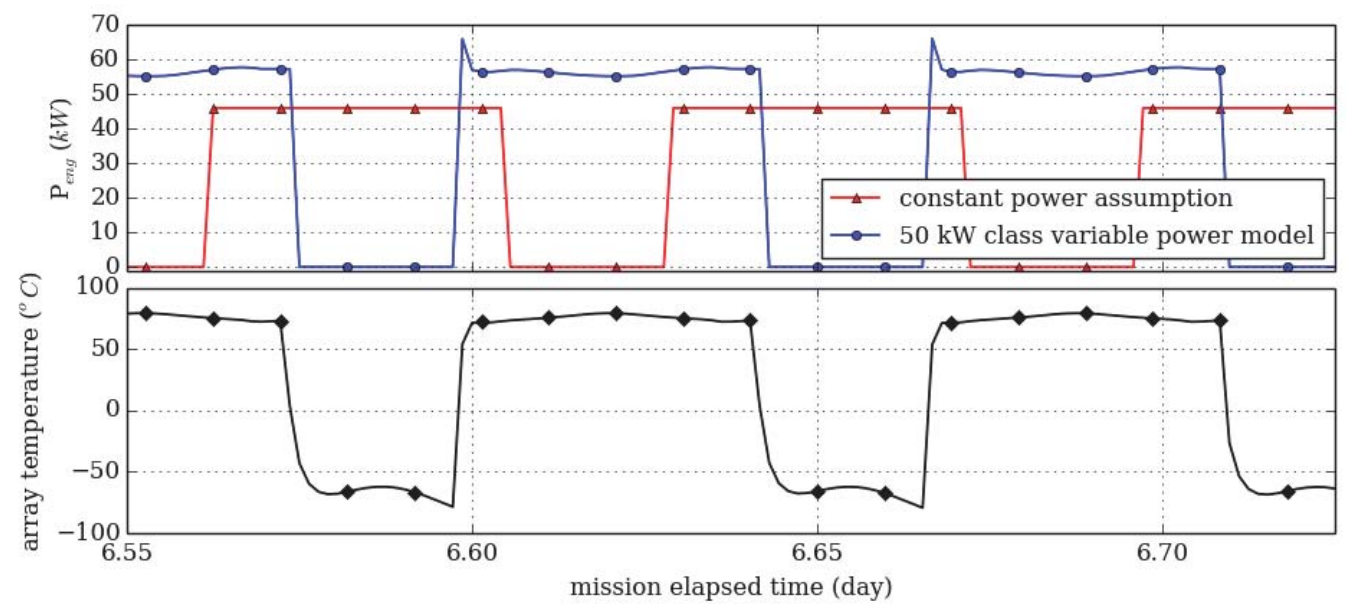

Figure 5: Time history $P_{\text {eng }}(\mathrm{kW})$ and power array temperature $\left({ }^{\circ} \mathrm{C}\right)$ for the $50 \mathrm{~kW}$ variable power case to illustrate the impact of the thermal model on power generation.

\section{B. Problem 2: Perfect Pointing Versus Roll Hold}

The spiral out problems in this paper follow pitch and yaw profiles generated by the DAG module in SEPSim's GN\&C suite; however, roll is determined by a separate algorithm. The variable power case from the previous section (Section III.A) is simulated with a "perfect pointing" algorithm where it is assumed that the vehicle can be roll-oriented to maximize array exposure to the sun at all times (RCS propellant usage is not modeled). The "perfect pointing" roll case from the previous section is compared to an assumed roll hold condition ( roll $=0 \mathrm{deg}$ ) in this section to illustrate how SEPSim can be used to investigate the interaction of GN\&C with the solar array model. Such comparisons are difficult to achieve without the use of an integrated tool such as SEPSim.

The resulting $P_{\text {eng }}$ profiles for the case detailed above are presented in Figure 6. For discussion purposes, $\beta_{\text {sun }}$ is defined to be the angle between the orbital plane of the vehicle and the corresponding Earth-Sun line. The pointing losses, associated with high $\beta_{\text {sun }}$ under the roll hold assumption, are clearly visible from the data and would, again, be information that could be incorporated in the mission design. Depending on the mission information, items such as the off-pointing losses may be absorbed if they occur early in the mission when the arrays are generating extra power due to oversizing. Alternatively, the mission design may need to plan ahead for off-pointing losses by including appropriate roll control measures to compensate for them; this is especially important if the mission is time critical.

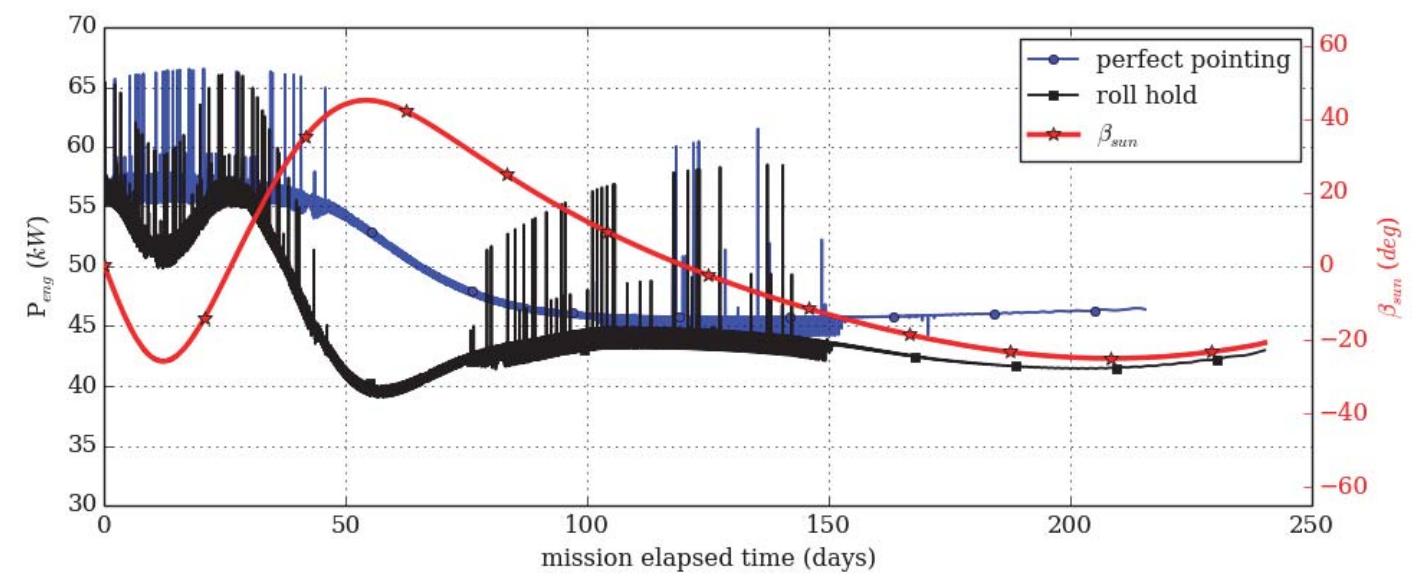

Figure 6: Time history of $P_{\text {eng }}(\mathrm{kW})$ for perfect pointing (blue) and with roll hold (roll=0) (black). $\beta_{\text {sun }}$ angle (deg) for roll hold case superimposed on right axes in red. 


\section{Conclusion}

An integrated tool for analyzing solar array performance during a high fidelity trajectory simulation has been developed for use in EP vehicle studies in the near Earth radiation environment. These studies are becoming increasingly important as NASA and other commercial entities become more interested in taking advantage of fuel savings that EP systems offer when sending payloads to high energy (e.g. geostationary or near escape) orbits. SEPSim offers researchers, mission, and vehicle and power system designers a unique capability to study their individual systems in an integrated simulation. The approach to developing SEPSim and some preliminary, lower-fidelity (i.e. 3DOF) results are summarized in this paper. The 3DOF results presented give an indication of the unique capability afforded to the research community through SEPSim's integrated approach. The tool allows the study of real-time array degradation, subsystem interactions and other effects along with their impact on mission design and solar array sizing. Previously, such studies have been difficult, of lower fidelity, time consuming and prone to error because of the decoupled nature of the process.

The work in this paper is meant to serve as a starting point for more advanced studies. The next logical progression for model development is to take advantage of the modularity of the tool to add higher fidelity models for propulsion and other pertinent subsystems. Finally, SEPSim, being built on the MASTIF platform, is capable of full $6 \mathrm{DOF}$ treatment for mature vehicle models.

\section{Acknowledgments}

The authors would like to thank Kevin E. Witzberger (NASA-ARC) who has been instrumental in the initial conceptual design and implementation of SEPSim. The authors would also like to thank Robert B. Klimek (NASA-GRC) for his continued support and code development towards the SEPSim project.

\section{References}

${ }^{1}$ Pederson, D. and Hojnicki, J., "Analysis of Roll Steering for Solar Electric Propulsion Missions," AIAA Paper AIAA2012-3890, American Institute of Aeronautics and Astronautics, July 2012, 48th Joint Propulsion Conference and Exhibit. draft.

${ }^{2}$ Witzberger, K., Smith, D., Martini, M., and Wright, T., MASTIF's User's Manual, NASA-GRC, 1st ed., 2011, working

${ }^{3}$ Hojnicki, J., Green, R., Kerslake, T., McKissock, D., and Trudell, J., "Space Station Freedom Electrical Performance Model," Tech. Rep. TM-1993-106395, NASA, August 1993, Presented at the 28th Intersociety Energy Conversion Engineering Conference, , Atlanta, Georgia.

${ }^{4}$ McCarter, J., Marshall Aerospace Vehicle Representation In 'C' (MAVERIC-II) User's Guide, NASA-MSFC, 2nd ed.

${ }^{5}$ Witzberger, K. and Smith, D., "Design and Assessment of Open-loop Variable Ignition Time Guidance for the Mars Ascent Vehicle," Tech. Rep. AAS 12-192, AAS/AIAA, January 2012, Presented at the 22nd AAS/AIAA Space Flight Mechanics Meeting, Charleston, South Carolina.

${ }^{6}$ Jannette, A., Hojnicki, J., McKissock, D., Fincannon, J., Kerslake, T., and Rodriguez, C., "Validation Of International Space Station Electrical Performance Model Via On-Orbit Telemetry," Tech. Rep. TM-2002-211803, NASA, July 2002, Presented at the 37th Intersociety Energy Conversion Engineering Conference, Washington, D.C.

${ }^{7}$ Hojnicki, J., Kerslake, T., Ayres, M., Han, A., and Adamson, A, M., "Adaptation and Re-Use of Spacecraft Power System Models for the Constellation Program," Tech. Rep. TM-1993-106395, NASA, July 2008, Presnted at the Sixth International Energy Conversion Engineering Conference, Cleveland, Ohio.

${ }^{8}$ Sawyer, D. M. and Vette, J. I., "AP-8 trapped proton environment for solar maximum and solar minimum," NASA STI/Recon Technical Report N, Vol. 77, December 1976, pp. 18983, Provided by the SAO/NASA Astrophysics Data System.

${ }^{9}$ Falck, R. D., Sjauw, W., and Smith, D., "Comparison of Low-Thrust Control Laws for Application in Planetocentric Space," Tech. Rep. submitted, AIAA, July 2014, To be presented at the 50th AIAA/ASME/SAE/ASEE Joint Propulsion Conference, Cleveland, Ohio.

${ }^{10}$ Ortiz Longo, C. and Rickman, S., "Method for the Calculatoin of Spacecraft Umbra and Penumbra Shadow Terminator Points," Tech. Rep. TP-3547, NASA, 1995, Available from the NASA Center for AeroSpace Information, Linthicum Heights, Maryland. 